\title{
Analysis of Correlated MRC with Transmit Antenna Selection under $\eta-\mu$ Fading
}

\author{
Concepción Téllez-Labao, Juan P. Peña-Martín and Juan M. Romero-Jerez, Senior Member, IEEE
}

\begin{abstract}
A wireless link considering correlated Maximal Ratio Combining (MRC) and Transmit Antenna Selection (TAS) under the general $\eta-\mu$ fading is analyzed. Exact and asymptotic expressions for the cumulative distributing function (CDF) and probability density function (PDF) are derived. These new results are used to evaluate the outage capacity and the asymptotic average bit error rate (average BER) for different binary modulations. The obtained theoretical results are validated through Monte Carlo simulations.
\end{abstract}

Index Terms-Bit error rate, asymptotic analysis, generalized fading.

\section{INTRODUCTION}

A MONG the different possible Multiple Input - Multiple Output (MIMO) schemes, which employ multiple transmit and receive antennas, Transmit Antenna Selection (TAS) with Maximal Ratio Combining (MRC) at the receiver provides full diversity gain and reduces the implementation complexity by selecting the transmit antenna that maximizes the output SNR [1]. Thus, TAS/MRC is currently the focus of much attention by the research community, as it has been proposed as a part of present and future wireless communications systems, including multi-hop, spectrum-sharing and securecommunications systems (see, for example, [2]-[5] and the references therein).

Multi-antenna signal reception in a portable or handheld terminal will typically yield to signal correlation due to insufficient antenna separation, resulting in a performance degradation that needs to be quantified for a proper system design. In spite of the vast amount of literature dealing with TAS/MRC systems, few works consider receive antenna correlation, and results seem to be available only for correlated Rayleigh and Nakagami-m fading, e.g. [6]-[9].

The $\eta-\mu$ fading is a general fading model that can be used to better represent the small-scale variations of the radio signal in a non-line-of-sight condition [10], and includes the Hoyt (Nakagami- $q$ ), the Nakagami- $m$ and the Rayleigh fading models as particular cases. On the other hand, the generality of the model yields to a considerable increased mathematical complexity, which precludes the derivation of simple expressions for the performance analysis of wireless

Copyright (c) 2017 IEEE. Personal use of this material is permitted. However, permission to use this material for any other purposes must be obtained from the IEEE by sending a request to pubs-permissions@ieee.org.

This work was supported by the European Regional Development Fund and the Spanish Ministry of Economy and Competitiveness under Grant TEC201342711-R.

The authors are with the Department of Electronic Technology, E.T.S.I. Telecomunicación, Universidad de Málaga, 29071 Málaga, Spain (e-mail: tellez@uma.es,.jppena@uma.es,romero@dte.uma.es). communication systems. Thus, to the author's knowledge, there is no previous result for TAS/MRC in correlated $\eta-\mu$ fading, and results seem to be available only for the case of uncorrelated antennas [11]. A general framework for the asymptotic evaluation of the symbol error rate (SER) suitable for arbitrary fading was presented in [2], however, uncorrelated receive antennas was assumed.

A comprehensive analysis of TAS/MRC under $\eta$ - $\mu$ considering arbitrary receive antenna correlation is presented in this work. The CDF and PDF of the instantaneous output signal-to-noise ratio (SNR) are derived both in exact form, in terms of a confluent Lauricella function, and in asymptotic form, which tends to the exact values as the average SNR per receive antenna increases. These chief statistics are new in the literature, to the authors' knowledge, and are used to find exact and asymptotic expressions of the outage capacity, as well as asymptotic BER expressions for several binary modulations.

The remainder of this work is organized as follows: The system model is presented in Section II. In Section III, exact and asymptotic expressions of the CDF and PDF of the instantaneous SNR are derived. In Section IV, expressions for the outage capacity and the asymptotic BER for several binary modulations are found. Some application results are shown in Section V. Finally, main conclusions are drawn in Section VI.

\section{SYSTEM MODEL}

We consider a MIMO system with $L_{r}$ receive antennas and $L_{t}$ transmit antennas. Spatial diversity is exploited at the transmit end by means of TAS, while at the receive end MRC is performed. We assume a slowly varying (with respect to the symbol duration) and non-selective (flat) fading. The transmit antennas are assumed to be uncorrelated, while the receive antennas are assumed to be spatially correlated with arbitrary correlation between any pair of antennas. An errorfree feedback channel is assumed to allow the transmitter to select the transmit antenna which maximizes the output SNR.

The channel between any transmit and receive antenna is assumed to undergo $\eta$ - $\mu$ fading. This is a general fading model that considers the received signal to be the composition of $2 \mu$ signal clusters, when $2 \mu$ is an integer, which is assumed here. Format 1 of this fading model is considered, in which the $\eta$ parameter represents the power ratio between the inphase and quadrature components in each signal cluster [10]. The instantaneous SNR at receive antenna $i$ conditioned on antenna $j$ being selected for transmission will be

$$
\gamma_{i \mid j}=\sum_{k=1}^{2 \mu_{i, j}} X_{i, k \mid j}^{2}+Y_{i, k \mid j}^{2}
$$


where $X_{i, k \mid j}$ and $Y_{i, k \mid j}$ are mutually independent zero-mean Gaussian random variables with variances $\sigma_{X_{i, k \mid j}}^{2}=\bar{\gamma}_{i \mid j} \eta_{i, j} /\left(2 \mu_{i, j}\left(1+\eta_{i, j}\right)\right)$ and $\sigma_{Y_{i, k \mid j}}^{2}=$ $\bar{\gamma}_{i \mid j} /\left(2 \mu_{i, j}\left(1+\eta_{i, j}\right)\right)$, where $\bar{\gamma}_{i \mid j}$ denotes the average SNR at receive antenna $i$ conditioned on antenna $j$ being selected for transmission, while $\eta_{i, j}$ and $\mu_{i, j}$ are the fading parameters in the link between transmit antenna $j$ and receive antenna $i$. The SNR at the output of the MRC combiner, conditioned on antenna $j$ being selected for transmission is given by

$$
\gamma_{j}=\sum_{i=1}^{L_{r}} \gamma_{i \mid j}
$$

It is proved in [12] that, when $2 \mu_{i . j}$ are integers, the MGF of $\gamma_{j}$ for correlated MRC reception under $\eta-\mu$ fading is given by

$$
\mathcal{M}_{\gamma_{j}}(s)=\prod_{v=1}^{V_{j}}\left(1-2 \lambda_{v}^{\left(X_{j}\right)} s\right)^{-\frac{\xi_{v}^{\left(X_{j}\right)}}{2}}\left(1-2 \lambda_{v}^{\left(Y_{j}\right)} s\right)^{-\frac{\xi_{v}^{\left(Y_{j}\right)}}{2}}
$$

where $\left\{\lambda_{v}^{\left(X_{j}\right)}\right\}_{v=1}^{V_{j}}$ and $\left\{\lambda_{v}^{\left(Y_{j}\right)}\right\}_{v=1}^{V_{j}}$ are the distinct eigenvalues, and $\left\{\xi_{v}^{\left(\bar{X}_{j}\right)}\right\}_{v=1}^{V_{j}}$ and $\left\{\xi_{v}^{\left(Y_{j}\right)}\right\}_{v=1}^{V_{j}}$ their associated algebraic multiplicities, of the covariance matrices $\mathbf{K}_{\mathbf{X}_{\mathbf{j}}}=$ $\operatorname{Cov}\left(\mathbf{X}_{\mathbf{j}}^{\mathbf{T}}, \mathbf{X}_{\mathbf{j}}\right)$ and $\mathbf{K}_{\mathbf{Y}_{\mathbf{j}}}=\operatorname{Cov}\left(\mathbf{Y}_{\mathbf{j}}^{\mathbf{T}}, \mathbf{Y}_{\mathbf{j}}\right)$, where $\mathbf{X}_{\mathbf{j}}=$ $\left[\mathbf{X}_{\mathbf{1} \mid \mathbf{j}}, \ldots, \mathbf{X}_{\mathbf{L}_{\mathbf{r}} \mid \mathbf{j}}\right]$ with $\mathbf{X}_{\mathbf{i} \mid \mathbf{j}}=\left[X_{i, 1 \mid j}, \ldots, X_{i, 2 \mu_{i, j} \mid j}\right]$, and $\mathbf{Y}_{\mathbf{j}}=\left[\mathbf{Y}_{\mathbf{1} \mid \mathbf{j}}, \ldots, \mathbf{Y}_{\mathbf{L}_{\mathbf{r}} \mid \mathbf{j}}\right]$ with $\mathbf{Y}_{\mathbf{i} \mid \mathbf{j}}=\left[Y_{i, 1 \mid j}, \ldots, Y_{i, 2 \mu_{i, j} \mid j}\right]$. Note that $\mathbf{K}_{\mathbf{X}_{\mathbf{j}}}$ and $\mathbf{K}_{\mathbf{Y}_{\mathbf{j}}}$ are square matrices of dimension $\sum_{i=1}^{L_{r}} 2 \mu_{i, j}$ and their elements are determined considering $\operatorname{Cov}\left(X_{i, k \mid j}, X_{p, q \mid j}\right)=\sigma_{X_{i, k \mid j}} \sigma_{X_{p, q \mid j}} \sqrt{\rho_{i, p}}$ and $\operatorname{Cov}\left(Y_{i, k \mid j}, Y_{p, q \mid j}\right)=\sigma_{Y_{i, k \mid j}} \sigma_{Y_{p, q \mid j}} \sqrt{\rho_{i, p}}$ if $k=q$, where $\rho_{i, p}$ is the correlation coefficient between the SNR at receive antennas $i$ and $p$. while $\operatorname{Cov}\left(X_{i, k \mid j}, X_{p, q \mid j}\right)=$ $\operatorname{Cov}\left(Y_{i, k \mid j}, Y_{p, q \mid j}\right)=0$ if $k \neq q$, with $i, p=1, \ldots, L_{r}$, $k=1, \ldots, 2 \mu_{i, j}$ and $q=1, \ldots, 2 \mu_{p, j}$.

For the sake of compactness, in the following, and unless explicitly stated otherwise, we will consider equal channel parameters for any pair of transmit and receive antennas, and the sub-indices will be dropped from the notation when unnecessary. In this case, the MGF given in (3) can be written in terms of the eigenvalues of the signal correlation matrix of the received antennas [13], which permits to avoid constructing matrices $\mathbf{K}_{\mathbf{X}}$ and $\mathbf{K}_{\mathbf{Y}}$. Actually, we demonstrate in the next Proposition that, in this case, the sum of correlated $\eta-\mu$ random variables is statistically equivalent to the sum of independent $\eta-\mu$ random variables where the individuals SNRs, assumed to be the same at every receive antenna, are weighted by the eigenvalues of the signal correlation matrix.

Proposition 1. Let us assume a correlated MRC receiver under $\eta$ - $\mu$ fading with equal parameters at every branch, then, the MGF of the output SNR, given that antenna $j$ is selected for transmission, can be written as

$$
\begin{aligned}
M_{\gamma_{j}}(s)= & \prod_{v=1}^{V}\left(1-\frac{\eta \bar{\gamma}}{\mu(1+\eta)} \lambda_{v} s\right)^{-\mu \zeta_{v}} \\
& \times\left(1-\frac{\bar{\gamma}}{\mu(1+\eta)} \lambda_{v} s\right)^{-\mu \zeta_{v}}
\end{aligned}
$$

where $\left\{\lambda_{v}\right\}_{v=1}^{V}$ are the distinct eigenvalues, and $\left\{\zeta_{v}\right\}_{v=1}^{V}$ their associated algebraic multiplicities, of the signal correlation matrix $\mathrm{M}$ of the receive antenna array, which is assumed to be non-singular. Note that $\mathbf{M}$ is a square matrix of dimension $L_{r}$ and $\sum_{v=1}^{V} \zeta_{v}=L_{r}$.

Proof: When the fading parameters at the receive antennas are equal, matrices $\mathbf{K}_{\mathbf{X}}$ and $\mathbf{K}_{\mathbf{Y}}$ are constructed considering that

$$
\begin{gathered}
\operatorname{Cov}\left(X_{i, k \mid j}, X_{p, q \mid j}\right)=\eta \operatorname{Cov}\left(Y_{i, k \mid j}, Y_{p, q \mid j}\right), \\
\operatorname{Cov}\left(Y_{i, k \mid j}, Y_{p, q \mid j}\right)=\left\{\begin{array}{l}
\frac{\bar{\gamma}}{2 \mu\left(\frac{1}{\gamma}+\eta\right)}, \text { for } i=p, k=q, \\
\frac{2 \mu(1+\eta)}{\rho_{i, p}}, \text { for } i \neq p, k=q, \\
0, \text { for the rest of cases, }
\end{array}\right.
\end{gathered}
$$

where $i, p=1, \ldots, L_{r} ; k, q=1, \ldots, 2 \mu$; and where $\rho_{i, p}$ is the correlation coefficient between the received SNR at antennas $i$ and $p$ of the receive array. Thus, the correlation matrices $\mathbf{K}_{\mathbf{X}}$ and $\mathbf{K}_{\mathbf{Y}}$ can be expressed in compact form as

$$
\begin{gathered}
\mathbf{K}_{\mathbf{X}}=\eta \mathbf{K}_{\mathbf{Y}}, \\
\mathbf{K}_{\mathbf{Y}}=\frac{\bar{\gamma}}{\mathbf{2 \mu}(\mathbf{1}+\eta)} \mathbf{M} \otimes \mathbf{I}_{\mathbf{2} \mu},
\end{gathered}
$$

where $\mathbf{I}_{n}$ denotes the $n \times n$ identity matrix, $\otimes$ denotes the kronecker product and $\mathbf{M}$ is the signal correlation matrix which elements are given by

$$
\mathbf{M}(i, p)=\sqrt{\rho_{i, p}}, \quad i, p=1, \ldots, L_{r} .
$$

From (7) and (8) and the fact that the eigenvalues of the kronecker product of two matrices are given by the product of the eigenvalues of the involved matrices, it is clear that the eigenvalues of $\mathbf{K}_{\mathbf{X}}$ and $\mathbf{K}_{\mathbf{Y}}\left(\lambda_{v}^{(X)}\right.$ and $\left.\lambda_{v}^{(Y)}\right)$ are related to the eigenvalues of $\mathbf{M}$ by

$$
\lambda_{v}^{(X)}=\frac{\bar{\gamma} \eta}{2 \mu(1+\eta)} \lambda_{v}, \quad \lambda_{v}^{(Y)}=\frac{\bar{\gamma}}{2 \mu(1+\eta)} \lambda_{v},
$$

which implies that $\mathbf{K}_{\mathbf{X}}, \mathbf{K}_{\mathbf{Y}}$ and $\mathbf{M}$ has the same number of different eigenvalues, satisfying $\xi_{v}^{(X)}=\xi_{v}^{(Y)}=2 \mu \zeta_{v}$, as $\mathbf{I}_{2 \mu}$ has a unity eigenvalue with multiplicity $2 \mu$, which together with (3) yields to (4).

Note that for $2 \mu=m, \eta=1$, the result in (4) collapses to [9, eq. (2)] for Nakagami- $m$ fading.

As TAS is performed at the transmitter, the unconditional output SNR will be given by $\gamma=\max _{j}\left\{\gamma_{j}\right\}, j=1, \ldots, L_{t}$. In the next section we derive both exact and asymptotic expressions for the PDF and CDF of $\gamma$.

\section{OUTPUT SNR STATISTICS}

We now show that it is possible to find exact expressions for both the PDF and the CDF of the output SNR of the proposed system model.

Lemma 1. The PDF and CDF of the output SNR of a correlated MRC receiver with transmit antenna selection under $\eta-\mu$ fading with equal parameters can be expressed in terms of the confluent Lauricella function $\Phi_{2}(\cdot)$, defined in $[14, p .34,(8)]$, as given, respectively, in (11) and (12), where we have defined $\mathbf{Z}=\left\{\zeta_{1}, \ldots, \zeta_{V}\right\}$ and $\boldsymbol{\Delta}=\mu(1+\eta)\left\{\lambda_{1}^{-1}, \ldots, \lambda_{V}^{-1}\right\}$. 


$$
\begin{gathered}
f_{\gamma}(x)=\frac{2 \mu L_{r} L_{t}}{\left[\left(2 \mu L_{r}\right) !\right]^{L_{t}}[\operatorname{det}(\mathbf{M})]^{2 \mu L_{t}}}\left(\frac{\mu^{2}(1+\eta)^{2}}{\eta}\right)^{\mu L_{r} L_{t}} \frac{1}{\bar{\gamma}}\left(\frac{x}{\bar{\gamma}}\right)^{2 \mu L_{r} L_{t}-1} \\
\times \Phi_{2}^{(2 V)}\left(\mu \mathbf{Z}, \mu \mathbf{Z} ; 2 \mu L_{r} ;-\frac{\boldsymbol{\Delta}}{\eta} \frac{x}{\bar{\gamma}},-\boldsymbol{\Delta}_{\bar{\gamma}}\right)\left[\Phi_{2}^{(2 V)}\left(\mu \mathbf{Z}, \mu \mathbf{Z} ; 2 \mu L_{r}+1 ;-\frac{\boldsymbol{\Delta}}{\eta} \frac{x}{\bar{\gamma}},-\boldsymbol{\Delta} \frac{x}{\bar{\gamma}}\right)\right]^{L_{t}-1}, \\
F_{\gamma}(x)=\frac{1}{\left[\left(2 \mu L_{r}\right) !\right]^{L_{t}}[\operatorname{det}(\mathbf{M})]^{2 \mu L_{t}}}\left(\frac{\mu^{2}(1+\eta)^{2} x^{2}}{\eta \bar{\gamma}^{2}}\right)^{\mu L_{r} L_{t}}\left[\Phi_{2}^{(2 V)}\left(\mu \mathbf{Z}, \mu \mathbf{Z} ; 2 \mu L_{r}+1 ;-\frac{\boldsymbol{\Delta}}{\eta} \frac{x}{\bar{\gamma}},-\boldsymbol{\Delta}_{\overline{\bar{\gamma}}}^{x}\right)\right]^{L_{t}} .
\end{gathered}
$$

Proof: From (4), the MGF of $\gamma_{j}$ can be rewritten as

$$
\begin{aligned}
& M_{\gamma_{j}}(s)=\frac{1}{[\operatorname{det}(\mathbf{M})]^{2 \mu}}\left(\frac{\mu^{2}(1+\eta)^{2}}{\eta \bar{\gamma}^{2}}\right)^{\mu L_{r}} \frac{1}{(-s)^{2 \mu L_{r}}} \\
& \times \prod_{v=1}^{V}\left(1-\frac{\mu(1+\eta)}{\eta \bar{\gamma} \lambda_{v} s}\right)^{-\mu \zeta_{v}}\left(1-\frac{\mu(1+\eta)}{\bar{\gamma} \lambda_{v} s}\right)^{-\mu \zeta_{v}}
\end{aligned}
$$

The PDF $f_{\gamma_{j}}(x)$ and $\mathrm{CDF} F_{\gamma_{j}}(x)$ of the output SNR conditioned on antenna $j$ being selected for transmission are given by the inverse Laplace transformations $f_{\gamma_{j}}(x)=$ $\mathcal{L}^{-1}\left[M_{\gamma_{j}}(-s) ; x\right]$, and $F_{\gamma_{j}}(x)=\mathcal{L}^{-1}\left[M_{\gamma_{j}}(-s) / s ; x\right]$, which can be found using [14, p. 290, (55)]. Considering now that the PDF and CDF of the output SNR are given, respectively, by $f_{\gamma}(x)=\sum_{j=1}^{L_{t}} f_{\gamma_{j}}(x) \prod_{k=1, k \neq j}^{L_{t}} F_{\gamma_{k}}(x)$ and $F_{\gamma}(x)=$ $\prod_{j=1}^{L_{t}} F_{\gamma_{j}}(x)$, the final results are obtained.

Remark. For the case of non-necessarily equal channel parameters between the transmit and receive antenna pairs, the $M G F$ of $\gamma_{j}$ given in (3) can be rewritten as

$$
\begin{aligned}
& M_{\gamma_{j}}(x)=\frac{1}{(-2 s)^{\sum_{i=1}^{L r} 2 \mu_{i, j}} \sqrt{\operatorname{det}\left(\mathbf{K}_{\mathbf{X}_{\mathbf{j}}}\right) \operatorname{det}\left(\mathbf{K}_{\left.\mathbf{Y}_{\mathbf{j}}\right)}\right.}} \\
& \times \prod_{v=1}^{V_{j}}\left(1-\frac{1}{2 \lambda_{v}^{\left(X_{j}\right)} s}\right)^{-\frac{\xi_{v}^{(X)}}{2}}\left(1-\frac{1}{2 \lambda_{v}^{\left(Y_{j}\right)} s}\right)^{-\frac{\xi_{v}^{\left(Y_{j}\right)}}{2}},
\end{aligned}
$$

and following the same approach as in Lemma 1, the PDF and CDF of the output SNR can be obtained for the general case. However, the resulting expressions will be less compact and not explicitly given in terms of the channel parameters and the average SNR per receive antenna, but in terms of matrices $\mathbf{K}_{\mathbf{X}_{\mathbf{j}}}$, $\mathbf{K}_{\mathbf{Y}_{\mathbf{j}}}$ and their eigenvalues.

The derived exact expressions can be accurately calculated numerically, however, they do not offer insights on the impact of system parameters on performance. Fortunately, they permit the derivation of asymptotic expressions in the high SNR regime.

Corollary 1. The asymptotic behavior, as $\bar{\gamma} \rightarrow \infty$, of the PDF and CDF of the output SNR of a correlated MRC receiver with transmit antenna selection under $\eta$ - $\mu$ fading with equal parameters can be obtained as

$$
\begin{aligned}
& f_{\gamma_{j}}(x)=\frac{2 \mu L_{r} L_{t}}{\left[\left(2 \mu L_{r}\right) !\right]^{L_{t}}[\operatorname{det}(\mathbf{M})]^{2 \mu L_{t}}} \\
& \times\left(\frac{\mu^{2}(1+\eta)^{2}}{\eta \bar{\gamma}^{2}}\right)^{\mu L_{r} L_{t}} x^{2 \mu L_{r} L_{t}-1}+o\left(\bar{\gamma}^{-2 \mu L_{r} L_{t}}\right), \\
& F_{\gamma_{j}}(x)=\frac{1}{\left[\left(2 \mu L_{r}\right) !\right]^{L_{t}}[\operatorname{det}(\mathbf{M})]^{2 \mu L_{t}}} \\
& \times\left(\frac{\mu^{2}(1+\eta)^{2}}{\eta}\right)^{\mu L_{r} L_{t}}\left(\frac{x}{\bar{\gamma}}\right)^{2 \mu L_{r} L_{t}}+o\left(\bar{\gamma}^{-2 \mu L_{r} L_{t}}\right),
\end{aligned}
$$

where the function $o(g(x))$ is any function such that $\lim _{x \rightarrow 0} o(g(x)) / g(x)=0$.

Proof: These expressions can be obtained from (11) and (12) and the fact that, from the definition of $\Phi_{2}(\cdot)$, given in [14, p. $34,(8)]$, it is clear that. $\Phi_{2}^{(n)}\left(b_{1}, \ldots, b_{1} ; c ; d_{1} x, \ldots, d_{n} x\right)=$ $1+o\left(x^{\epsilon}\right)$, with $0<\epsilon<1$.

Note that the obtained PDF has the form

$$
f_{\gamma}(x) \doteq \frac{\alpha}{\bar{\gamma}}\left(\frac{x}{\bar{\gamma}}\right)^{t}+o\left(\bar{\gamma}^{-(t+1)}\right)
$$

where parameters $t$ and $\alpha$ are given by

$$
\begin{gathered}
t=2 \mu L_{r} L_{t}-1, \\
\alpha=\frac{2 \mu L_{r} L_{t}}{\left[\left(2 \mu L_{r}\right) !\right]^{L_{t}}[\operatorname{det}(\mathbf{M})]^{2 \mu L_{t}}}\left(\frac{\mu^{2}(1+\eta)^{2}}{\eta}\right)^{\mu L_{r} L_{t}} .
\end{gathered}
$$

Note that parameters $\alpha$ and $t$ can be used to readily obtain closed-form expressions of different performance metrics, such as the average error rate for different binary and $M$-ary modulations in the high SNR regime [15].

\section{Performance analysis of Wireless COMMUNICATIONS SYSTEMS}

As we have been able to obtain exact and asymptotic expressions of the correlated MRC receiver with TAS under $\eta-\mu$ fading, we can calculate different performance metrics of wireless communications systems employing this MIMO scheme under the considered fading model. As an example of application, we compute the outage capacity probability and the asymptotic BER for several binary modulations. 


\section{A. Outage capacity probability}

The instantaneous channel capacity per unit bandwidth is well known to be given by

$$
C=\log _{2}(1+\gamma) .
$$

We define the outage capacity probability as the probability that the instantaneous channel capacity $C$ falls below a predefined threshold $R_{S}$ (given in terms of rate per unit bandwidth), i.e.,

$$
P_{\text {out }}=P\left(C<R_{S}\right)=P\left(\log _{2}(1+\gamma)<R_{S}\right) .
$$

Therefore

$$
P_{\text {out }}=P\left(\gamma<2^{R_{S}}-1\right),
$$

yielding

$$
P_{\text {out }}=F_{\gamma}\left(2^{R_{S}}-1\right),
$$

which together with (12) permits to calculate the exact outage capacity probability. This result offers little insight on the effect of parameters on performance. Fortunately, we can obtain a simple expression in the high SNR regime as, from (16), we can write

$$
\begin{aligned}
P_{\text {out }} & \doteq \frac{1}{\left[\left(2 \mu L_{r}\right) !\right]^{L_{t}}[\operatorname{det}(\mathbf{M})]^{2 \mu L_{t}}} \\
& \times\left(\frac{\mu^{2}(1+\eta)^{2}}{\eta}\right)^{\mu L_{r} L_{t}}\left(\frac{2^{R_{S}}-1}{\bar{\gamma}}\right)^{2 \mu L_{r} L_{t}}, \quad \bar{\gamma} \rightarrow \infty,
\end{aligned}
$$

where the symbol $\doteq$ denotes asymptotic equality as $\bar{\gamma} \rightarrow \infty$.

\section{B. Asymptotic average BER}

The average error rate for several binary modulations can be expressed in compact form using [16, eq. (8.100)] as

$$
\overline{P_{e}}=\int_{0}^{\infty} \frac{\Gamma(b, a x)}{2 \Gamma(b)} f_{\gamma}(x) d x,
$$

where $\Gamma(m, x)=\int_{x}^{\infty} z^{m-1} e^{-z} d z$ denotes the incomplete Gamma function, $\Gamma(m)=\Gamma(m, 0)$ denotes the Gamma function and where $(a, b)=(1,0.5)$ for binary phase shift keying (BPSK), $(a, b)=(0.5,0.5)$ for binary frequency shift keying (BFSK), $(a, b)=(1,1)$ for differential binary phase shift keying (DBPSK) and $(a, b)=(0.5,1)$ for non-coherent binary frequency shift keying (NCBFSK). Alternatively, integrating (25) by parts, the average error rate can be computed from the $\mathrm{CDF}$ as

$$
\overline{P_{e}}=\frac{a^{b}}{2 \Gamma(b)} \int_{0}^{\infty} x^{b-1} e^{-a x} F_{\gamma}(x) d x .
$$

From the derived exact expression of the CDF given in (12) it is not possible to find a closed-form exact expression for the average BER. However, (26) is well suited for numerical evaluation using the Gauss-Laguerre quadrature method [17, p. 890 (25.4.45)]. On the other hand, the introduction of (16) into (26) leads to a closed-form asymptotic expression as

$$
\begin{aligned}
\overline{P_{e}} \doteq & \frac{\Gamma\left(b+2 \mu L_{r} L_{t}\right)}{2 \Gamma(b)\left[\left(2 \mu L_{r}\right) !\right]^{L_{t}}[\operatorname{det}(\mathbf{M})]^{2 \mu L_{t}}} \\
& \times\left(\frac{\mu^{2}(1+\eta)^{2}}{\eta}\right)^{\mu L_{r} L_{t}} \frac{1}{(a \bar{\gamma})^{2 \mu L_{r} L_{t}}}, \quad \bar{\gamma} \rightarrow \infty .
\end{aligned}
$$

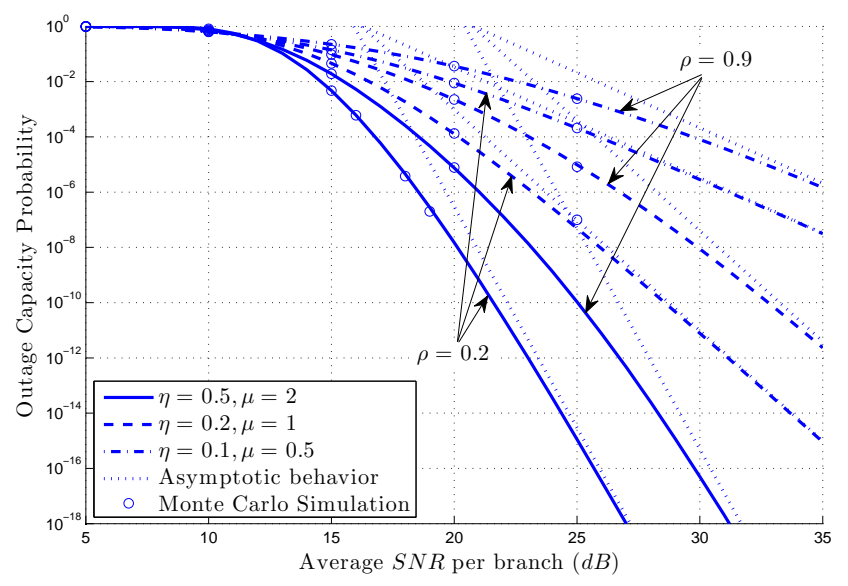

Figure 1. Outage capacity probability vs. average SNR per branch for a capacity threshold $R_{S}=5 \mathrm{bps} / \mathrm{Hz}$ when $L_{r}=2$ and $L_{t}=2$.

\section{NUMERICAL RESULTS}

In this section, we show some representative results of the analyzed system. For simplicity of discussion, we have considered a constant correlation $\rho$ between each pair of receive antennas. The $\Phi_{2}(\cdot)$ function is fast and efficiently computed as a numerical Inverse Laplace Transform using [18].

Fig. 1 shows the outage capacity probability vs. the average SNR per branch for a given capacity threshold $R_{S}=5 \mathrm{bps} / \mathrm{Hz}$ when $L_{r}=2$ and $L_{t}=2$ considering correlated receive antennas. The figure plots the exact results, the asymptotic results and also some points obtained by Monte Carlo simulation that show an excellent agreement with the theoretical results. We can appreciate how, for a given set of values for the channel parameters $\eta$ and $\mu$, the higher the correlation the greater the outage probability, as expected due to the loss of channel variability among the receive antennas. We can also see in Fig. 1 the influence of the channel parameters: the severity of fading decreases when $\mu$ increases and, consequently, the outage probability becomes lower. On the other hand, the $\eta$ parameter has a much smaller influence and it can be observed that the higher the value of $\eta$, for $\eta \in(0,1]$, the lower the fading severity, thus yielding a better performance.

Fig. 2 represents the outage capacity probability vs. correlation coefficient for a capacity threshold $R_{S}=5 \mathrm{bps} / \mathrm{Hz}$ and $\bar{\gamma}=15 \mathrm{~dB}$. The influence of the sizes of the transmit and receive arrays with different channel parameter values can be analyzed. It is shown that, as the number of antennas increases, the outage capacity probability reduces. Comparing the cases, $L_{r}=2, L_{t}=3$ and $L_{r}=3, L_{t}=2$, it is interesting to note that a better performance (lower outage capacity probability) is obtained when more antennas are deployed at the receiver, except for the case of high correlation, as the MRC receiver is less effective when the receive antennas experience similar channels.

Finally, Fig. 3 shows the asymptotic average BER for different modulation schemes, correlation and channel parameter values. As expected, the average asymptotic BER increases 


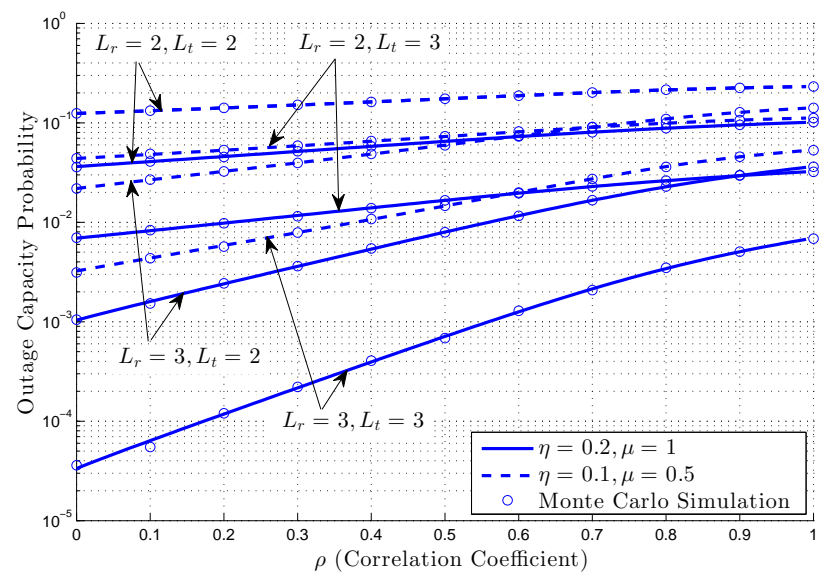

Figure 2. Outage capacity probability vs. correlation coefficient for a capacity threshold $R_{S}=5 \mathrm{bps} / \mathrm{Hz}$ and an average SNR $\bar{\gamma}=15 \mathrm{~dB}$.

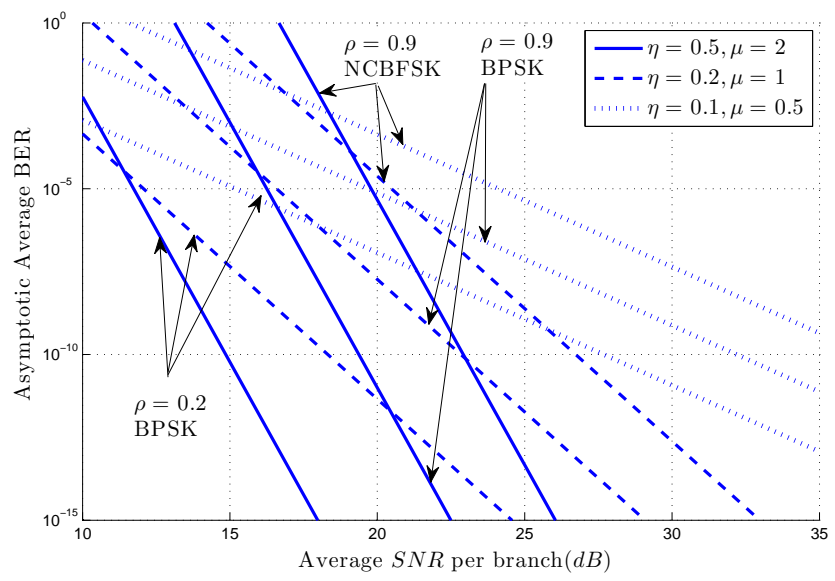

Figure 3. Asymptotic average BER vs. average SNR per branch for $L_{r}=2$ and $L_{t}=2$..

when the channel fading is more severe (lower $\eta$ and/or $\mu$ ). On the other hand, it is shown how correlation degrades performance, i.e., the lower $\rho$ the lower the BER in the high SNR regime. Also, it is shown that the NCBFSK modulation shows a worse behavior than BPSK, as the former is a noncoherent modulation.

\section{CONCLUSIONS}

In this work, expressions for the PDF and CDF of the output SNR are derived for the first time for TAS/MRC systems under $\eta-\mu$ fading and arbitrary correlation at the receive end, both in exact and asymptotic form. As application examples, we have used these expressions to analyze the outage capacity probability and the asymptotic average BER for different binary modulations. The asymptotic expressions allow a much affordable analysis in the high SNR regime. Monte Carlo simulation shows an excellent agreement with the theoretical results.

The framework presented here can be used to analyze more elaborated system models for which the TAS/MRC scheme has been proposed, such as multi-hop, spectrum-sharing and secure-communications systems, but incorporating the general $\eta-\mu$ fading with receive antenna correlation.

\section{REFERENCES}

[1] S. Thoen, L. Van der Perre, B. Gyselinckx, and M. Engels, "Performance analysis of combined transmit-SC/receive-MRC," IEEE Trans. Commun., vol. 49, no. 1, pp. 5-8, Jan. 2001.

[2] P. L. Yeoh, M. Elkashlan, N. Yang, D. B. da Costa, and T. Q. Duong, "Unified Analysis of Transmit Antenna Selection in MIMO Multirelay Networks," IEEE Trans. Veh. Technol., vol. 62, no. 2, pp. 933-939, Feb. 2013.

[3] P. L. Yeoh, M. Elkashlan, K. J. Kim, T. Q. Duong, and G. K. Karagiannidis, "Transmit Antenna Selection in Cognitive MIMO Relaying With Multiple Primary Transceivers," IEEE Trans. Veh. Technol., vol. 65, no. 1, pp. 483-489, Jan. 2016.

[4] D. C. Gonzalez, D. B. da Costa, and J. C. S. Santos Filho, "Distributed TAS/MRC and TAS/SC Schemes for Fixed-Gain AF Systems With Multiantenna Relay: Outage Performance," IEEE Trans. Wireless Commun., vol. 15 , no. 6, pp. 4380-4392, Jun. 2016.

[5] J. Xiong, Y. Tang, D. Ma, P. Xiao, and K.-K. Wong, "Secrecy Performance Analysis for TAS-MRC System With Imperfect Feedback," IEEE Trans. Inf. Forensics Security, vol. 10, no. 8, pp. 1617-1629, Aug. 2015.

[6] B.-Y. Wang and W. X. Zheng, "Outage probability of transmitter antenna selection/receiver- MRC over spatially correlated Nakagamifading channel," in Signal Processing, 2006 8th International Conference on, vol. 3, 16-20 2006.

[7] B.-Y. Wang and W.-X. Zheng, "BER performance of transmitter antenna selection/receiver-MRC over arbitrarily correlated fading channels," IEEE Trans. Veh. Technol., vol. 58, no. 6, pp. 3088 -3092, Jul. 2009.

[8] B.-Y. Wang, "Accurate BER of transmitter antenna selection/receiverMRC over arbitrarily correlated Nakagami fading channels," in Acoustics, Speech and Signal Processing, 2006. ICASSP 2006 Proceedings. 2006 IEEE International Conference on, vol. 4, may 2006, p. IV.

[9] J. P. Peña Martín, C. Tellez-Labao, and J. M. Romero-Jerez, "Impact of fading severity and receive antenna correlation on TAS/MRC under Nakagami fading," in Proc. 2016 International Conference on Computing, Networking and Communications (ICNC), Feb. 2016.

[10] M. D. Yacoub, "The $\kappa-\mu$ distribution and the $\eta-\mu$ distribution," IEEE Antennas Propag. Mag., vol. 49, no. 1, pp. 68-81, Feb. 2007.

[11] B. Kumbhani and R. S. Kshetrimayum, "Analysis of TAS/MRC based MIMO Systems over $\eta-\mu$ Fading Channels," IETE Technical Review, vol. 32, no. 4, pp. 252-259, Jul. 2015.

[12] V. Asghari, D. da Costa, and S. Aissa, "Symbol Error Probability of Rectangular QAM in MRC Systems With Correlated $\eta-\mu$ Fading Channels," IEEE Trans. Veh. Technol., vol. 59, no. 3, pp. 1497-1503, Mar. 2010.

[13] D. Morales-Jimenez, J. F. Paris, and A. Lozano, "Outage Probability Analysis for MRC in $\eta-\mu$ Fading Channels with Co-Channel Interference," IEEE Communications Letters, vol. 16, no. 5, pp. 674-677, May 2012.

[14] H. Srivastava and P. Karlsson, Multiple Gaussian hypergeometric series. Ellis Horwood Ltd., 1985.

[15] Z. Wang and G. Giannakis, "A simple and general parameterization quantifying performance in fading channels," IEEE Trans. Commun., vol. 51, no. 8, pp. 1389-1398, Aug. 2003.

[16] M. K. Simon and M.-S. Alouini, Digital Communications over Fading Channels, 2nd ed., ser. Wiley-Interscience. John Wiley \& Sons, Inc., 2005.

[17] M. Abramowitz and I. A. Stegun, Handbook of Mathematical Function s with Formulas, Graphs, and Mathematical Tables, 10th ed. U.S. Department of Commerce - N.B.S., Dec. 1972.

[18] E. Martos-Naya, J. M. Romero-Jerez, F. J. Lopez-Martinez, and J. F. Paris, "A MATLAB program for the computation of the confluent hypergeometric function $\Phi_{2}$, , Technical Report, Universidad de Malaga, 2016. [Online]. Available: http://hdl.handle.net/10630/12068 